\title{
The MINWii Project: Renarcissization of Patients Suffering from Alzheimer's Disease Through Video Game-Based Music Therapy
}

\author{
Samuel Benveniste ${ }^{1}$, Pierre Jouvelot ${ }^{1}$, and Renaud Péquignot ${ }^{2}$ \\ ${ }^{1}$ CRI MINES ParisTech, 35, rue Saint-Honoré, 77305 Fontainebleau, France \\ Tel/Fax: +33164694708 \\ \{samuel . benveniste, pierre . jouvelot\}@mines-paristech.fr \\ ${ }^{2}$ Hôpital Saint-Maurice, 14, rue Val d'Osne, 94415 Saint-Maurice Cedex, France \\ Tel/Fax: +33143966363 \\ r.pequignot@hopital-saint-maurice.fr
}

\begin{abstract}
MINWii, a new serious video game targeting Alzheimer and demented patients, is a simple Music Therapy tool usable by untrained care givers. Its objective is to improve patients' self-image (renarcissization) to reduce behavioral symptoms, which are an important cause of institutionalization. With MINWii, elderly gamers use Wiimotes to improvise or play predefined songs on a virtual keyboard. We detail our design process, which addresses the specific features of dementia: this iterative refinement scheme, built upon qualitative, small scale experiments in a therapeutic environment, led to a shift of MINWii's original focus from creativity to reminiscence. A large majority of our patients, with mild to moderate dementia, expressed a strong interest in our system, which was confirmed by feedback from the care givers. A fully controlled usability study of MINWii is currently under way, which should lead to future research assessing its actual therapeutic impact.
\end{abstract}

Keywords: Alzheimer, Dementia, Music Therapy, Wiimote, Renarcissization.

\section{Introduction}

According to the World Health Organization [20, more than 18 million people worldwide suffer from Alzheimer's disease, the most common form of dementia. Researchers estimate that this pandemic may easily escalate to affect 1 in 85 people in 2050 6], 70\% of whom in developed countries. Caring for so many dependant patients already costs trillions of dollars annually [20, and remains rather uneffective. Indeed, the best that current drugs can do, in most cases, is to slightly slow down the cognitive decline.

Consequently, until satisfying treatment methods are found, our aging societies will have to adapt and attend to the needs of an ever-growing number of demented patients; non-pharmaceutical approaches can help with this issue [2]. 
Among them, two techniques seem to stand out [13]: Cognitive Stimulation Therapy (CST) [18] and the one of interest here, namely Music Therapy (MT).

MT techniques fall into two broad categories: Receptive MT, where patients listen to musical pieces under the supervision of therapists, and Active MT (AMT), where patients actually compose and perform music. Since patients treated with MT often lack any musical training and cannot properly play anything beyond simple percussions, elaborate computer systems such as Hyperscore from MIT Media Lab [5] or Riley's musical touchscreen [16] have been designed to address this issue, although their use has yet to spread in the MT community.

Here, we experiment with a new approach: given the surprising success of Nintendo's Wii with the elderly [21, we borrow techniques and off-the-shelf components such as the Wiimote from the world of video games to empower MT practitioners with a low-cost yet versatile tool, as was done in previous work [4, albeit for a very different condition (ADHD). To create MINWii, where patients use Wiimotes to either improvise music or play predefined songs on a virtual keyboard displayed on screen, we took the specific features of Alzheimer's disease into account when designing the gameplay. We then performed a qualitative, small scale usability test in a therapeutic environment to iteratively improve our design. We show here that the system is indeed usable by care givers with no musical training dealing with patients with mild to moderate impairment; moreover, a large majority of our test patients expressed a strong interest in MINWii, which was further confirmed by feedback from the care givers.

In Section 2, we explain our renarcissization-based approach through the use of video games and MT. We motivate the key design choices we made in MINWii in Section 3. low cognitive and motor HMI requirements, failure-free gameplay and use of off-the-shelf components. We describe in Section 4 our two-phase experimental validation process, where we test our first design, implement subsequent upgrades and show how the final version helps patients improve their self-image. We conclude and introduce future work in Section 5.

\section{Renarcissization for Dementia}

When used in the context of dementia treatment, renarcissization [1] refers to the process of restoring self-esteem in patients who have gradually grown to consider themselves as a useless burden for their care givers. Our approach gives them the opportunity to engage into activities specifically designed to highlight and make use of whatever capabilities they may still have.

\subsection{Behavioral Issues}

Besides reducing patients' depression levels, renarcissization could have a significant economic impact since, in an overwhelming majority of cases, behavioral issues are one of the main causes of institutionalization, often against the patients' will. Indeed, they frequently try to prove to their care givers that they can still live their life as usual, failing to realize the extent of their disability. They 
might for example routinely take their shower with their clothes on or completely mess up their home while trying to clean, exhausting their care givers. Worse, they might jeopardize their own safety and that of others by imprudence, eventually leading their family to give up and send them to a hospital or an institution, where they are more likely to be depressed and die faster [19].

On the contrary, patients with higher self-esteem are less likely to try to do things they cannot do anymore. A large part of them, who are otherwise healthy enough to stay at home, therefore require only minimal daily care such as food delivery and help for personal hygiene. This has great benefits in terms of cost: a visit from a nurse and a few hours of help from a personal assistant every day cost roughly half as much as a permanent stay in a caring home and almost three times less than a stay at the hospital.

Thus, there is a dire need for affordable and easy-to-use tools that untrained helpers such as family members could use to reduce or even prevent the onset of behavioral disorders in demented patients with minimal equipment and training costs. We expect that the blending of MT and video games within MINWii can (1) be instrumental in making demented patients feel better and (2) ease the time-consuming, psychologically-demanding burden of caring for them on their assistants and families.

\subsection{Music Therapy and Video Games}

The rationale for using MT in the treatment of Alzheimer's disease is as simple as it is compelling: for a large majority of patients, musical sensitivity, in particular regarding songs discovered during their childhood, is one of the very last cognitive capabilities to disappear. Moreover, it has been shown that techniques such as U-Cycle Receptive MT 9] efficiently reduce the anxiety levels of mild to moderately incapacitated patients. Thus, given its power and universality, music appears to be a good potential vector for the renarcissization of demented patients.

However, incorporating AMT in the treatment of dementia can be difficult: (1) there is a lack of music therapists even though demand is growing thanks to influential supporters such as Oliver Sacks 17] and (2) patients usually cannot use elaborate instruments. As a result, traditional AMT sessions, though in fact therapeutically effective, often end up as somewhat unappealing to patients and their family, with the therapist doing most if not all of the playing.

We think using a video game-based approach can alleviate at least some of these problems:

- By building on the success of the Wii in elderly care institutions 21] and providing data logging for easy assessment of patients' progress, we intend to convince geriatricians to consider and support the use of AMT;

- By providing ways for non-MT practitioners to use AMT in virtually any setting, including the patient's home, since MINWii simply requires a computer and a TV, we hope to help establishing AMT as a standard practice in dementia treatment and, hopefully, encourage institutions to go one step further and hire a real music therapist; 
- By offering a virtually infinite library of customizable instruments and interfaces, we aim at helping AMT become more enticing and patient-specific.

\section{Designing for Dementia}

Demented patients' skills deteriorate at surprisingly different rates. Some simple aptitudes such as the ability to remember a few objects may be long gone before seemingly higher-level ones such as singing a song begin to decline. Therefore, a straightforward simplification of a regular music video game like Guitar Hero 10 . would probably not be an effective music therapy tool. We suggest below that the playing experience can be made much more enjoyable for the patients if, instead, we manage to build upon the specific abilities they still have while keeping very low requirements in the areas where they are diminished. In this section we describe the key design points we established for the first version of our system: low cognitive and motor requirements, a rewarding user experience and hardware, software and operational simplicity.

\subsection{Low Cognitive and Motor Requirements}

Conditions such as arthritis and motor coordination deficits prevent elderly patients from making large or fast movements involving too many joints. Therefore our design emphasizes pointing using Wiimotes: only wrist movements within most patients' zone of comfort in terms of amplitude are required. Furthermore, MINWii heavily filters Wiimote inputs and cursor movements so that the pointer behaves smoothly enough for the patients to feel comfortable. Finally, to make it easier to hold the Wiimote, we added the possibility to use a Wii Pistol: this hollow plastic gun, in which the Wiimote can be encased, enables playing without having to bend the wrist in a way that might be uncomfortable. Regarding cognitive issues, pointing has a low footprint, since it is very intuitive: the user does not need to learn a new convention such as "this button does this" or "this arrow does that". Moreover, the Wii Pistol obviously has a great affordance for this task, though it has the potential downside of diverting the focus of the game from music to shooting.

To keep lowering the cognitive weight of our system, we designed an extremely simple, image-based graphical interface. We were very careful to adapt the visual part of the application to our population's reduced attention and focus abilities, often complemented with some degree of visual impairment. Everything on the screen is very large and highly contrasted to be easy to comprehend and we kept the eye candy to a minimum to avoid distracting users with unnecessary frills.

More to the point, we also tried to accommodate for the great decision-making difficulties demented patients usually have. The buttons on the Wiimote all do exactly the same thing and there is always one and only one active area at a time, highlighted as clearly as possible. This way, with only one method of interaction and only one object to interact with, the user never has a choice to make once the game has started and is much less at risk of feeling lost. Interestingly, this 


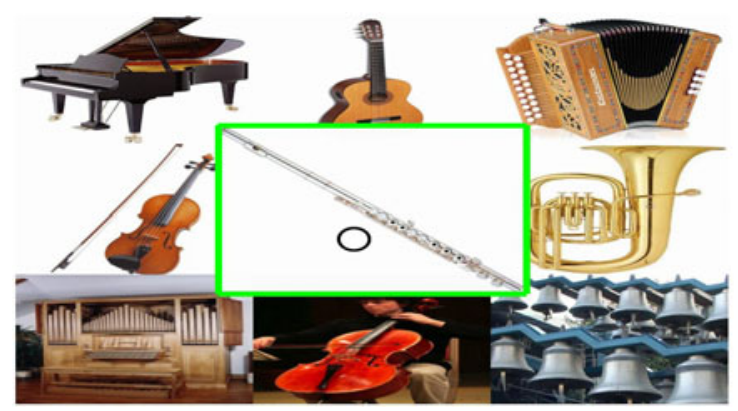

Fig. 1. Instrument choice screen

feature is at the opposite of what is usually considered as a key gameplay asset in traditional video games; the challenge here is to shrink the choice space while keeping the fun factor as high as possible.

Finally, to make the system look easy to use, we chose light pastel colors to get a nice soothing look, since it has been shown that a beautiful interface actually is easier to use [11]. Also, all the configuration settings (game difficulty, Wiimote sensitivity, pointing mode,...) are made by the care giver with his/her own Wiimote with no graphical output; indeed, we observed that visual indications related to such abstract notions often confuse demented patients and might induce anxiety by making the system look too complicated (see below).

\subsection{Failure-Free Gameplay}

Demented patients often exhibit a systematically defensive attitude regarding anything unfamiliar. They are always afraid to fail or break something when confronted with a new task. They will also give up very easily if they do not succeed right away, even when receiving strong encouragements from the care givers.

For such reasons, we designed a gameplay where failure is either impossible or very unlikely due to the simplicity of the task at hand. Moreover, if it does happen, its importance is systematically toned down. First, no click will result in a dissonant sound since only notes guaranteed to sound well together can be played during the game (e.g., a $\mathrm{C}$ major scale). Second, given how difficult it is for demented patients to use almost any electronic device autonomously whatever the design efforts invested [16], we prefer to see our system as a tool to foster interaction between patients and care givers. MINWii does not present judgments of any kind (scoring, game over, etc.) to the patient and relies on the care giver to both give praise and help through failure.

\subsection{Simplicity in Design}

As stated before, we have chosen to rely exclusively on off-the-shelf components in order to build a system that could be installed pretty much anywhere for less 
than $\$ 1000$, which is roughly the average price of a one-day hospital stay. The only necessary equipment is: a standard computer $(\$ 500)$, a computer-enabled TV screen $(\$ 400)$, a wireless infra-red Sensor Bar $(\$ 20)$ to be placed below the screen and at least one Wiimote $(\$ 40)$. We did use commercial sound synthesis PC software for prototyping, but the next version of MINWii, expected to be soon used in a large scale test, will rely exclusively on free software (pygame [15], fluidsynth [7] etc.) under the GNU Public License. Note that all the interactions can be logged for subsequent analysis. Though it is not required, we suggest using a personal USB thumb drive $(\$ 15)$ for each patient, containing his/her prefered configuration, logs and scores throughout the treatment, as is done at InGame Lab 3 .

Off-the-shelf components like the Wiimote have another interesting characteristic, less obvious but probably even more relevant: they usually have a simple, pleasant aesthetic design. For demented patients who are typically over 80 years old, this is of importance since any apparent complexity such as lots of wires, strange-looking machines etc. may trigger their constant fear of failing and breaking objects. The experience of one of us with personal hygiene robots [14] showed that even carefully designed, very easy to use devices can be of little practical interest with some patients because of that. MINWii only uses two pieces of nowadays familiar equipment (laptop and TV) and remote-like devices (Wiimotes and Sensor Bar); it is thus much less likely to be rejected for this kind of reasons.

\subsection{MINWii 0.9}

The initial version of MINWii we designed included only the Improvisation and Challenge Modes. Both can be played with any of the nine instruments available; note that we could have included many more and chose to offer only nine just to keep the pictures big enough on the screen (Figure 1).

Improvisation Mode. Here, patients are invited to improvise using a scale of their choice (e.g. C major) by pointing at a virtual keyboard of 8 or 11 colored keys displayed on the screen, depending on their level (determined by the care giver). A large white dot shows where they are aiming at and highly contrasts with the keys in the background, which all have their unique pastel color and display the name of their note (in French). The closer to the top of the screen one clicks, the higher the volume is (see Figure 2). Though it is hard to play fast, rather elaborate melodies can be played quite expressively at a slow tempo. However the temptation to speed up can be great, so we added a glissando option that can be switched on or off by the care giver; this allows for more "experimental" improvisations, but makes it much more difficult to play expressively.

Challenge Mode. Here, the patient has to choose a song to play. We started with the songs that patients tend to remember the most, hoping it would encourage them to play: simple nursery rhymes that most French children are familiar 


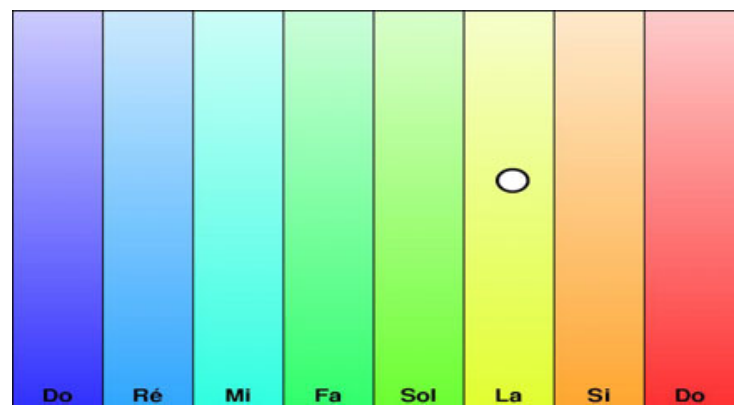

Fig. 2. Improvisation screen

with. This is a general rule with Alzheimer's disease: childhood memories tend to remain longer than more recent ones and therefore will grow in importance as patients forget the rest.

In this mode, the patient clicks the keys that highlight successively according to the song chosen. If the player hits a wrong key, the corresponding note is played, but at a lower volume. Since all the notes belong to the same scale, this will usually not sound too bad and can even allow the player to add a little personal touch to the song if he/she wants.

\section{Testing and Results}

We used a protocol akin to Action Research [12 to test MINWii and freeze the final design of our system while already helping patients at the same time. By putting these often lonely people at the center of the design process and making them feel useful by asking them for opinions, giving them praise or simply thanking them, we hoped to compensate for engaging them in an activity that we had yet to prove to be beneficial and that could even conceivably have been detrimental to their well-being, however unlikely that seemed to us at the time.

\subsection{Population and Protocol}

MINWii primarily targets demented patients with mild to moderate cognitive impairment, and potentially more severe (but not profound) difficulties in the visual, auditive and motor areas. We tested our system in the Geriatrics Unit of Hôpital Saint-Maurice (France), where patients usually stay for up to two months, depending on their condition and the follow-up care available.

We selected 9 patients with suspicion of Alzheimer's disease, a Mini Mental State score 8 between 10 and 25 (out of 30) and no profound deafness, blindness or motor disabilities. From November 2009 to February 2010, once a week, available patientswere encouraged to come to the animation room and "play music with the remotes". We usually ended up with a group of 3 or 4 whom we would sit in a semi-circle in front of the projection screen. They would each be given a 
Wiimote and then would take turns playing the song of their choice, with staff members sitting next to them and giving as much help as needed.

\subsection{A Need for Guidance}

For the very first session, only the Improvisation Mode was available. We were thrilled to see that patients were willing to play when asked to, some of them even showing signs of great enthusiasm and rather surprising pointing skills for a first trial. However, none of them were willing to explore the system in depth on their own, let alone improvise. When invited to do so, they said things like "I don't know any music" or "Hum me some tunes" and never even tried to click random notes, shake the Wiimotes or click the corners of the screen the way most of the healthy subjects who tried the application during informal testing did. However, when we suggested a song they remembered, they were more than happy to play it. Of course, even though one patient needed no help at all (she was a former pianist and had a rather high MMS rating of 22), the others were not able to play a song without extensive help from a musically trained care giver. Furthermore, although 8 or 11 notes are more than enough to improvise, it is hard to find many songs that fit into a single scale.

On the other hand, in the Challenge Mode we tested the following week, patients could play a song without needing help to find the right notes to click on. They clearly were more comfortable following the highlighted notes than trying to create something on their own. We felt we were on the right track because patients would now clap, sing and start reminiscing more and more often. We thus decided to leave the Improvisation Mode as is, and use it only with particularly skilled patients such as the one mentioned above. Interestingly, although this patient was barely able to keep things in memory for more than a couple of minutes, she complained that the playable songs were the same from one week to the other, which greatly surprised the physician among us.

However, in Challenge Mode, failure is rather likely due to the relatively small size of the keys on the screen. Thus, playing the song from start to end demanded quite a lot of time and became rather frustrating without active physical help from a care giver: patients had a lot of trouble following even simple rhythms on their own and were thus unable to recognize the songs. One specific quote illustrates this fact well: when leaving after a session, one patient said "It's hard: we have to remember the songs to sing". This caught our attention because even though this patient had quite a lot of trouble playing in Challenge Mode, what was perceived as hard was not playing, but remembering the song, which was exactly what we had tried to simplify.

We got a lot of similar feedback which showed that patients either (1) are not really aware of how much help with manipulating the Wiimotes they are given when they play or simply (2) do not care about properly pointing and clicking; in any case, they did not seem to mind that the care giver was actually doing almost of the Wiimote handling for them. What they did notice however is whether they were able to remember the melody and lyrics or not. Consequently, we decided to tone down the pointing challenge to an extremely low level and 
concentrate on the memory aspects of the activity which appeared to have a greater impact on their self-image.

\subsection{The Power of Reminiscence}

To focus the gameplay on reminiscence, we lowered the game difficulty to a level well below what we had intended in the beginning. First, in Reminiscence Mode (see Figure 3), the highlighted key is three times as big as the others, occupying more than a fourth of the screen, and displays the corresponding lyrics in large letters. Second, instead of having to click in the right place to play, patients can simply hover over the highlighted notes with the trigger pressed in, and the system makes sure that the melody is played with the proper rhythm. One can thus play without even looking at the screen by simply pushing the trigger and shaking the Wiimote from left to right, something that one of our test subjects, even though severely disabled, was thrilled to discover with some practice.

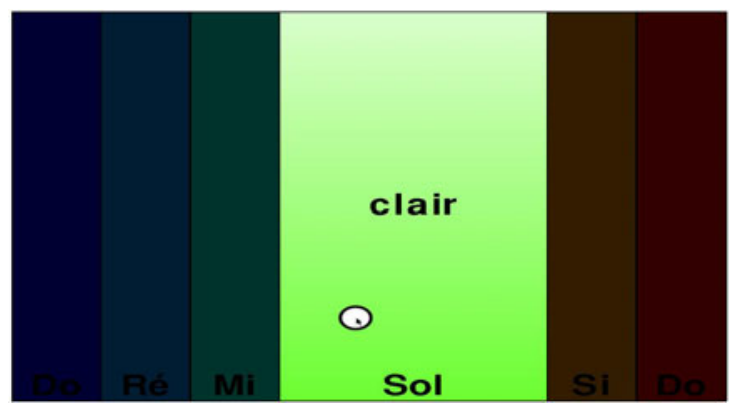

Fig. 3. "Au clair de la lune" in Reminiscence Mode

In fact, we were worried that such an easy task might deter our test subjects by making them feel underestimated. We were completely wrong: none of the patients ever said the task was too easy. Even elderly people without any signs of dementia who happened to pass by during our sessions seemed rather proud to be able to play a song despite the simplicity of the task. We think this is because, for people who were born in the 1920s, any task involving computers is often seen as utterly complicated. Therefore, being able to do even the simplest thing with something so "otherworldly" as a Wiimote is considered an achievement.

This observation is crucial: no matter how easy we make the game, patients will still feel empowered when playing it. We can therefore really tailor our system to trigger as much reminiscence as possible without having to worry about the gameplay becoming dull. This is very important because patients are usually well aware that they forget things more and more rapidly over time and often seem ecstatic when they discover that they do remember the songs from their childhood. Furthermore, the musical memories that come back often trigger a train of other flashbacks (actual quotes: "My husband was a trumpet player; 
he often played this when we went to the ball; these were happy times" or "We sung this at X's wedding; how much fun we had back then!").

These recollections usually seem to be even more pleasurable to the patients than the actual singing and can sometimes lead to signs of completely unexpected awareness. For instance, one severely disabled patient (MMS $=10$ ) told us, after playing a song by a famous French singer a few weeks in a row, that she was sad because he was very sick at the time, maybe about to die. The care givers were astonished that a patient with such a high cognitive deficit was able to understand and retain such an information. Thus, even though it could seem like a good idea to design MINWii with the intent of making it playable autonomously, the need to smooth out the impact of such highly emotional occurrences justifies the presence of care givers while the game is being played. Yet, this anecdote also illustrates the tremendous potential of music therapy, which can have unanticipated, very profound cognitive effects [17, far beyond its usually accepted benefits regarding anxiety relief and memory stimulation [13.

\subsection{Putting Human Contact First}

Our last key design point is human interaction: MINWii is tailored to encourage patients to interact with care givers, family and other patients. Indeed, patients told us praises such as "You're all so nice: it's a pleasure coming here", "Thank you for singing with me: you're a much better singer than I am" or even "For people as lonely as I, human contact is very precious", which led us to reconsider our system not only as a game, but also as a medium for interaction.

First, we never tried to make MINWii usable by patients alone: a care giver always manages the overall progress of the game. For instance, the various songs available need to be presented to the patient in an understandable way. Thus, we decided to implement an accessible file browser with two main characteristics: (1) fonts are very big and all the unnecessary information and buttons are hidden in order not to confuse patients and (2) the titles of the songs are displayed in lieu of their filenames so that patients can read "Oh When The Saints" instead of "ohwhenthesaints.musicxml". This dialog screen is much more important than we had thought at first: demented patients retain their reading ability very long into their illness, to a point that many of our test subjects would read aloud whatever was written on the screen, often without a clue about what it meant. Therefore, just like realizing that they can still remember and sing a song, being able to read and understand written words with the help of the care givers is very pleasing for them, and is a great occasion to interact and give praise.

Second, MINWii's very flexible gameplay, which can be adjusted from quite a serious challenge to an extremely simple task, gives it the ability to appeal to many audiences besides demented patients. More specifically, several informal tests have convinced us that, if children visit, playing with MINWii could be a lot of fun for them and would constitute a very good way to encourage interaction with the patients, mainly through singing and clapping. And anyone who has observed elderly patients can tell that hardly anything could top the pleasure they take in spending some quality time with their great grandchildren. 
However, many patients rarely get visits, and the people they see the most are those treated in the same institutions, be it in day-care or long stay units. Just like other people, they chat, take walks and develop friendships, but their illness does make things more difficult, as they have trouble remembering faces and having coherent discussions. Thus, to enhance human interaction, we chose to organize our sessions in the hospital animation room with groups of 3 or 4 patients in front of a big projection screen. Note that, given how easy it is to set MINWii up, it would have been much easier for us, but overall less effective, to plan one-to-one private sessions since demented patients, who have a blurred perception of time, are often quite reluctant to leave their room for fear of missing a visit or a meal, even though nothing is up for them in the next 4 hours.

Our experiment showed that we were indeed able to have patients cooperate in musical production. Since Alzheimer's disease greatly hinders patients' multitasking ability, it is rather hard for the person playing to sing the song at the same time, even though the two tasks are very easy if taken separately. Having group sessions made it possible to still have the patients sing by encouraging an "I play, you sing" dynamic in the group. We were happy to see patients discuss musical preferences, clap and congratulate each other after playing or even, in a few cases, encourage other, more reluctant patients to come to the sessions and play music like "cowboys", as one patient explained because of the pistol.

\section{Conclusion}

Even though Music Therapy has been shown to be an effective approach to deal with demented patients, few easily usable systems specifically target them. Our MINWii platform, where patients use Wiimotes to either improvise music or play predefined songs on a virtual keyboard, was designed with the specific features of Alzheimer's disease and dementia in mind. Using an iterative process built upon qualitative, small scale experiments in a therapeutic environment, we ended up with a final design that focuses less on creativity but more on reminiscence and renarcissization, which appear to be strong motivators for our test population. A large majority of our patients, with mild to moderate dementia, expressed a strong interest in our system, which was further confirmed by feedback from the care givers, who were able to use MINWii even with no prior musical training.

We are currently undertaking a fully controlled usability study of MINWii, which, if successful, will lead to future research intending to assess its therapeutic impact. A long term goal of our research is also to study the possible use of the interaction data recording facilities of MINWii for applications in evidence-based medicine such as the patient-specific quantitative assessment of Music Therapy compared to other techniques.

\section{Acknowledgments}

We thank the patients for their feedback and enthusiasm, Pierrette Despres for her precious help and Nathalie Roy-Sanchez for making this study possible. 


\section{References}

1. Amyot, A.: Trois cas de figure sur la psychothérapie des personnes âgées. Psychothérapies 1(28), 70-132 (2008)

2. ANAES: Prise en charge non médicamenteuse de la maladie d'Alzheimer et des troubles apparentés (2003),

http://www.has-sante.fr/portail/upload/docs/application/
pdf/Alzheimer\%5Ftechno.pdf

3. Backlund, P., et al.: In: Game Lab, http://www.his.se/ingame

4. Benveniste, S., Jouvelot, P., Lecourt, E., Michel, R.: Designing Wiimprovisation for Mediation in Group Music Therapy with Children Suffering from Behavioral Disorders. In: Proceedings of the ACM Conference IDC 2009, pp. 18-26 (2009), http://www.cri.ensmp.fr/people/sbenven/MAWIIDCPaper.pdf

5. Boulanger, A.: Expressive Gesture Controller for an Individual with Quadriplegia. In: Proceedings of the 10th ACM Conference UbiComp 2008, pp. 113-116 (2008), http://opera.media.mit.edu/Current\%20website/ Boulanger\%5Fubicomp08.pdf

6. Brookmeyer, R., Johnson, E., Ziegler-Graham, K., Arrighi, H.M.: Forecasting the Global Burden of Alzheimer's Disease. Alzheimer's and Dementia 3.3, 186-191 (2007), http://works . bepress . com/rbrookmeyer/23

7. Fluidsynth, http://fluidsynth.resonance.org

8. Folstein, M.-F., Folstein, S.-E., McHugh, P.-R.: "Mini-mental state": A practical Method for Grading the Cognitive State of Patients for the Clinician. Journal of Psychiatric Research 12(3), 198-198 (1975)

9. Guetin, S., et al.: Effect of Music Therapy on Anxiety and Depression in Patients with Alzheimer's Type Dementia: a Randomised, Controlled Study. Dement. Geriatr. Cogn. Disord. 28(1), 36-46 (2009)

10. Guitar Hero, http://www.guitarhero.com

11. Hassenzahl, M.: The Interplay of Beauty, Goodness and Usability in Interactive Products. Human Computer Interaction 19, 319-349 (2004)

12. Lewin, K.: Group Decision and Social Change. Holt, Rinehart and Winston (1958)

13. Livingston, G., et al.: Systematic Review of Psychological Approaches to the Management of Neuropsychiatric Symptoms of Dementia. American Journal of Psychiatry 162(11), 1996-2021 (2005), http://ajp.psychiatryonline org/cgi/reprint/162/11/1996

14. Non-Invasive Continence Management System, http://people.brunel.ac.uk/\%7Eibsreav/NICMS/project.htm

15. Pygame Framework, http://www.pygame.org

16. Riley, P., Alm, N., Newell, A.: An interactive Tool to Promote Musical Creativity in People with Dementia. Computers in Human Behavior 25(3), 599-608 (2009)

17. Sacks, O.: Musicophilia, Tales of Music and the Brain. Vintage, New York (2008)

18. Spector, A., Thorgrimsen, L., Woods, B., Royan, L., Davies, S., Butterworth, M., Orrell, M.: Efficacy of an Evidence-Based Cognitive Stimulation Therapy Program for People with Dementia. The British Journal of Psychiatry 183, 248-254 (2003)

19. Tun, S.-M., et al.: Predictive Validity of Neuropsychiatric Subgroups on Nursing Home Placement and Survival in Patients with Alzheimer's Disease. Am. J. Geriatr. Psychiatry 15(4), 314-327 (2007)

20. Vas, C., et al.: Alzheimer's Disease, The Brain Killer. World Health Organization manual (2001), http://www.searo.who.int/LinkFiles/ Health\%5Fand $\% 5$ FBehaviour $\% 5$ Falzheimers.pdf

21. Yam, M.: Wii Invades Retirement Home, http://www. dailytech.com/Wii+Invades+Retirement+Home/article6191.htm 